\title{
Radiographic Signals Detection of Systemic Disease. Orthopantomographic Radiography
}

\author{
Detección de Signos Radiográficos en Enfermedades Sistémicas. Radiografía Ortopantomográfica \\ "Plauto Christopher Aranha Watanabe; **Allan Farman; \\ "Marlivia Gonçalves de Carvalho Watanabe \& *Joao Paulo Mardegan Issa
}

\begin{abstract}
WATANABE, P. C. A.; FARMAN, A.; WATANABE, M. G. D. C. \& ISSA, J. P. M. Radiographic signals detection of systemic disease. Orthopantomographic radiography. Int. J. Morphol., 26(4):915-926, 2008.

SUMMARY: For the purposes of this report, "systemic disease" will be interpreted as conditions that are spread out within the body rather than localized strictly to the tissues of the oral cavity. Since it would take many volumes to review all such conditions, the intent of the authors is to review a few examples of conditions where initial panoramic radiographic findings suggested widespread disease of significance enough to affect the quality of life and longevity of the patient.
\end{abstract}

KEY WORDS: Panoramic Radiography; Systemic disease.

\section{INTRODUCTION}

Panoramic radiography produces an image that includes both the maxillary and mandibular dental arches and the such surrounding structures as the maxillary antra, nasal fossa, temporomandibular joints, styloid processes, and hyoid bone. Although, dentists might concentrate only on the teeth and their supporting tissues when the examining panoramic radiographs, they should also be able to identify all other structures that appear in the image (White et al., 2004a; Farman et al., 1993; Watanabe et al., 2004).

The Selection of Patients for X-Ray Examination, US Food And Drug Administration Center for Devices and Radiological Health (FDA/CDRH) guidelines were first published in 1987, spurred by concern about the US population's total exposure to radiation from all sources. In 2004, updated guidelines were published following work by a joint panel of the American Dental Association and FDA (American Dental Association, 2004). The updated guidelines expanded use of panoramic examination as an alternate baseline dental radiographic examination, recognizing that panoramic technology has improved.
We can notice in the guide that the panoramic x-ray is used in the routine of all the types of patients. Its use also has endorsement of "Portaria 453" of the Health Department - ANVISA - Brazil, in the recommendation based on the principle of radioprotection "ALARA" (As Low As Reasonably Achievable), or either, we must always use the lesser possible amount of radiation for the attainment of diagnostic information of our patients (Secretaria da Vigilância Sanitária, 1998).

While panoramic radiograph should not be prescribed primarily for detection of non-maxillofacial conditions, it is incumbent upon the health practitioner to be cognizant of panoramic image features that are indicative of systemic health. For the purposes of this paper, "systemic disease" means conditions that are disseminated within the body rather than localized strictly to the tissues of the oral cavity. Since it would take many volumes to review all such conditions, the intent here is to present examples of conditions, where initial panoramic radiographic findings suggested widespread disease significant enough to affect the quality of life and longevity of the patient.

\footnotetext{
" University of São Paulo, Faculty of Dentistry of Ribeirão Preto, Department of Morphology, Stomatology and Physiology, Brazil.

** University of Louisville, School of Dentistry of Kentucky, Department of Radiology and Imaging Sciences, USA.
} 


\section{Systemic disease}

1. Osteoporosis. "Osteo" is Latin for "bone." "Porosis" means "porous or full of holes." Hence, "Osteoporosis" means "bones that are full of holes." Bone mass reflects the balance between formation by osteoblasts and resorption by osteoclasts. Around the third decade of life the peak bone mass is reached, then there starts a slow but steady loss of bone with increased age. Osteoporosis is a multifactorial metabolic bone disease characterized by low bone mineral density (BMD), the deterioration of the micro-architecture of cancellous bone, and changes in the physical properties of bone, leading to greater bone fragility with increased fracture risk. Risk factors for osteoporosis include increased age, female sex, genetics, environment (e.g. nutrition; physical activity; medication and smoking), and hormonal deficiency. BMD as measured by dual energy $x$-ray absorptiometry (DEXA) has become the primary way to assess the risk of fracture, even if it is only a surrogate measure of bone strength. Osteoporosis is diagnosed when the value for BMD is 2.5 standard deviations or more below the mean of the young adult reference range (World Health Organization criteria) (Wowern, 2001).

Osteoporosis can progress asymptomatically until a bone fractures. One in two women and one in eight men over the age of 50 years will develop osteoporosis. Osteoporosis is the cause of bone fractures and can lead to chronic back pain (most common), loss of physical height, protruding stomach, stooped posture. At age 50 years, a woman has a 50\% chance of an osteoporosisrelated fracture during the rest of her life. A woman's hip fracture risk equals her combined risk of breast, uterine and ovarian cancer. Fifteen to $20 \%$ need long-term care due to loss of ability to do daily living activities, such as cooking, cleaning, and getting dressed. Fifty per cent of people who suffer a fractured hip lose the ability to live independently. Around $20 \%$ of people who fracture a hip die within a year, as a result of their fracture (Secretaria da Saúde, 1995) (Fig. 1).

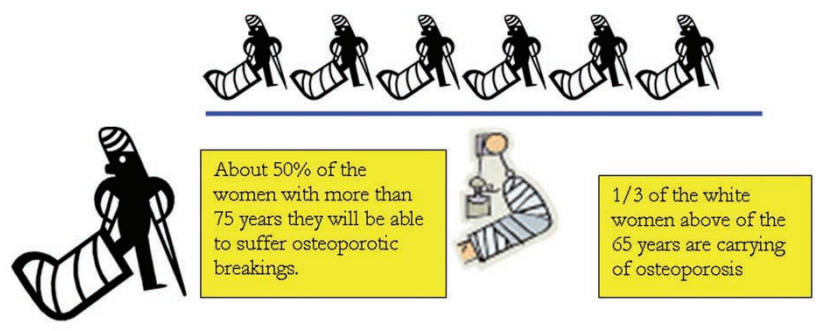

Fig. 1. Some data epidemiologics about osteoporosis in the world.
In Brazil, the prevalence of osteoporosis is little known, but it measured the use of resources and annual cost for patients with osteoporosis, pos-menopausal in the clinic of osteoporosis of the UNIFESP, the annual average cost for patient was approximately, \$ 442,00/patient. However, some authors (Araújo et al., 2006) assessed the direct cost during hospitalization for an osteoporotic hip fracture in Brazilian private health care system, by health plan companies' perspective, so the authors concluded that this cost had assess in approximately $\$ 6.900$. The study "Osteoporosis - Brazil Year 2000," developed by 300 medical specialists, estimated that less than a third of Brazilians with osteoporosis are diagnosed, and that only $20 \%$ of those known to be affected are treated (Marques Neto \& Lederman, 1995).

1.1.Radiographic features of osteoporosis. Cardinal radiographic factors of osteoporosis in the skeleton include generalized osteopenia, thinning and accentuation of the bone cortices, and accentuation of primary and loss of secondary trabeculation. Subordinated radiological factors include spontaneous, atraumatic fracture, especially of the spine, wrist, hip or hibs, basilar invagination in the skull and granular appearance of the bone in the skull (Wowern, 1986). Radiological features of osteoporosis in the jaws (Figs. 2 and 3) include relative radiolucency of both jaws and reduced definition of the cortices. An investigation of cross-sectional bone morphometric analyses in vitro (Wowern, 1986) has shown that the bone structure of the normal dentate jaws in older individuals is characterized by relatively thin porous cortical bone lamellae with endosteal demineralization, as in other bones, and these agerelated cortical changes tend to be more common in women than in men. In the jaws, the pronounced inter-individual and regional variations in the structure and density of cancellous bone can mask the sex and age-related decrease in demonstrated in other cancellous parts of the skeleton (Wowern, 1986). The methods for assessing age-related jaw bone changes in vivo were listed by Bras et al., 1982.
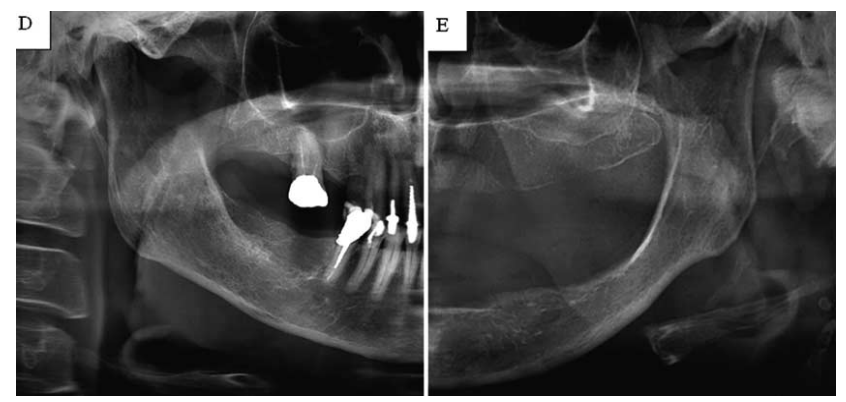

Fig. 2. Osteoporosis . Cropped panoramics images shows a relative radiolucency of both jaws with reduced definition and mandibular inferior cortex moderately eroded, evidence of lacunar resorption (right-D) or cortex severely eroded (left-E). 


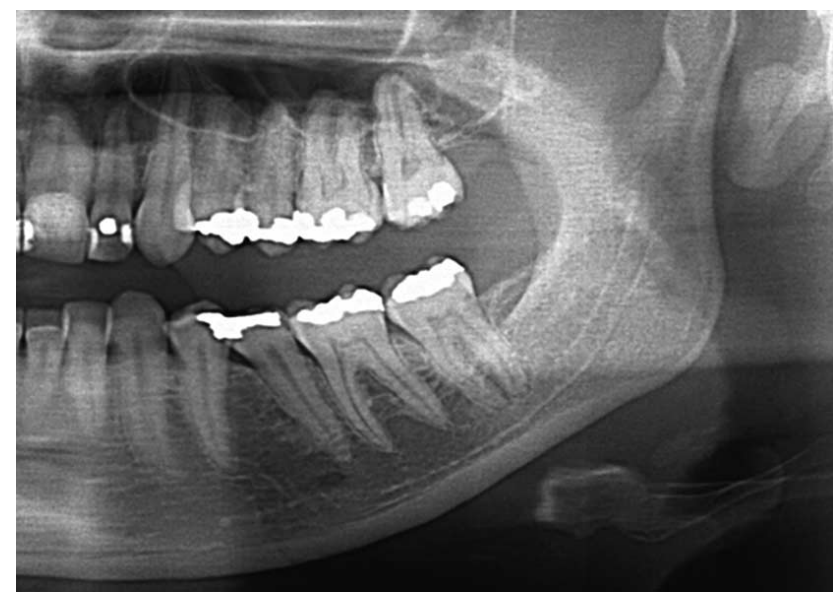

Fig. 3: Normal Mandibular inferior cortex - Cropped panoramics images.

1.2. Evidence supporting a role for panoramic radiography in screening for osteoporosis.In 1991, it was described a panoramic radiomorphometric index of mandibular cortical bone, the panoramic mandibular index (PMI). Differences in the index in a population of 353 adult subjects, equally divided by sex, age (30 through 79), and racial group (Black, Hispanic, White), were evaluated with respect to side, racial group, sex age, and combinations of these variables (Benson et al., 1991). Blacks were found to have a greater mean PMI than Hispanics or Whites, who were demographically similar. Age-related changes comparing younger and older age groups within each sex and racial group indicated a significant decrease in mean PMI with increasing age in black and Hispanic women.

A retrospect investigation was made to determine associations between spinal density and the density of selected mandibular sites as determined from panoramic radiographs in women between the ages of 50 and 75 years of known low bone density. The radiographs were randomized and then digitized for density analyzes. Significant differences were found between the groups at the $95^{\text {th }}$ percentile level. Hence, according to this study, it is possible to differentiate between persons of high and low mineral bone density using panoramic radiographs (Mohammad et al., 1996).

The literature concerning radiographic oral signs of osteoporosis was reviewed in 2002, including alveolar bone resorption, and reduction in cortical mandibular bone thickness. It was concluded that the panoramic radiograph is an important tool that to provide indications for the diagnosis of osteoporosis (Bulgarelli et al., 2002). Yet (Homer et al., 2002) was studied the relative usefulness of clinical and radiographic indices in the diagnosis of patients with low skeletal bone mass amongst 135 healthy peri-menopausal women, aged 45-55 years, attending for dental treatment. Bone mineral density was measured for the spine and femoral neck using DEXA and calculated according to the WHO criteria for Caucasian women. Each patient received a panoramic radiograph, and the width of the inferior mandibular cortex (MI) was measured. The body mass index (BMI) and simple calculated osteoporosis risk estimation (SCORE) indices were also calculated to help predict low bone mass, but the weight of the patient was the only significant constituent factor. MI, BMI and SCORE indices were significantly correlated with skeletal bone density. When the logistic regression model included MI, BMI and SCORE indices, all three variables were significant predictors of low skeletal bone mass. So, the authors could concluded that a thinning of the mandibular cortices ( $\mathrm{MI}<3 \mathrm{~mm}$ ) in normal peri-menopausal females is associated with low skeletal bone mass. If, in addition, the patient were underweight (BMI below $20 \mathrm{~kg} / \mathrm{m}^{2}$ ) or has a high SCORE index $(\geq 6)$ there was increased risk of osteoporosis (Homer et al.).

It was investigated (2003) General Dental Practitioners' (GDPs) agreement and diagnostic efficacy in detecting post-menopausal women with low BMD, from the appearance (normal or eroded) of the mandibular inferior cortex on panoramic radiographs (Nakamoto et al., 2003). The observers were 27 GDPs asked to classify MI appearance on panoramic radiographs of 100 post-menopausal women who had completed BMD assessments of the lumbar spine and of the femoral neck. Diagnostic efficacy (sensitivity, specificity and predictive values) was analyzed by comparing two groups classified by the MI (women with normal versus those having an eroded mandibular inferior cortex) with those classified by BMD (women with normal BMD versus women with osteopenia or osteoporosis). The mean sensitivity and specificity measures were $77 \%$ and $40 \%$, respectively, when BMD of the lumbar spine was the standard, and $75 \%$ and $39 \%$, respectively when BMD of the femoral neck was the standard. Nineteen untrained GDPs $(70 \%)$ presented a moderate to-almost perfect intra-observer agreement (Nakamoto et al.). It was concluded that the panoramic radiograph can be used in clinical dental practice to identify postmenopausal women, who have undetected low BMD and need to undergo further testing by bone densitometry (Nakamoto et al.; Taguchi et al., 2003).

Three indicators of bone quality on panoramic radiographs were studied (2003) for correlation with BMD using DEXA in brazilian people (Watanabe, 2003). A study of bone trabeculae and the mandible cortex in panoramic radiographs was found to reveal early signs of osteoporosis to the dentist. The thickness of the cortex and trabecular 
mandibular pattern in 58 panoramic radiographs and BMD were correlated. Statistical significance $(\mathrm{p} \leq 5 \%)$ were found in correlations between fractal dimension (FD), percentage of black pixels (ET) and connectivity, and significant correlation at the level $\mathrm{p} \leq 1 \%$ were proven for the MI, and also for FD and ET. There were significant correlations in relation to BMD, MI and cortical width. There was no significant correlation between the parameters analyzed (FD, ET and connectivity) and BMD.

The diagnostic performances of panoramic measurements (MI) and an osteoporosis self-assessment tool (OST) for identifying women with spinal osteoporosis (WHO criteria), in 159 healthy post-menopausal and 157 postmenopausal women with histories of hysterectomy, oophorectomy, or estrogen use were compared, in 2004 (Taguchi et al., 2004). MI shape and width were evaluated on panoramic radiographs. Receiver operating characteristic (ROC) curve analysis was used to determine the optimal cutoff thresholds for cortical width and the OST in healthy postmenopausal women. The authors concluded that the dentists may be able to refer postmenopausal women with suspected spinal osteoporosis for bone densitometry on the basis of dental panoramic radiographs with diagnostic performance similar to that of osteoporosis screening tools based on questionnaires. The correlation of the Classification of Klemetti et al., 1994, for MI using digital panoramic images of Brazilian women was studied in 2004 (Watanabe et al., 2004b). Correlations were made against forearm BMD accomplished by DEXA. Significant correlations were found between the two techniques, indicating that panoramic radiography has value in the determination of patients at risk for osteoporosis.

The BMD and linear radiomorphometric parameters of the mandible in elderly patients with different types of dentures were examined (Knezovic'-Zlataric' \& $\mathrm{C}^{\text {`elebic', }}$ 2005). Subjects had mandibular complete dentures (CDs) or Kennedy Class I removable partial dentures (RPDs) in the mandible. Three parameters were measured in panoramic radiographs (PRs) from a total of 136 subjects: thicknesses of the mandibular cortex below the mental foramen, the antegonion, and the gonion. Mandibular BMD was measured densitometrically from PRs using copper step wedge. The results showed that there was a significant difference between patients with mandibular CDs and those with mandibular RPDs for all the radiomorphometric indices measured ( $\mathrm{p}<$ 0.001). Specifically, radiomorphometric indices were higher in mandibular RPD wearers. Mandibular BMD values in mandibular RPD wearers were higher than those of patients with mandibular CDs, and BMD was significantly higher under the saddle in mandibular RPD wearers $(p<0.05)$. Yet in 2005 (White et al., 2005a) was suggested that the dentists possess clinical and radiographic information sufficient to detect patients with osteoporosis. White et al., 2005b concluded that changes in panoramic radiographic trabecular structure complemented by clinical information are predictive for hip fracture of aged women.

So, the physician must suspect of the risk of osteoporosis, when to find the following signals in the panoramic $x$-ray, in the jaws (Watanabe):

- Class II or III by Klemetti, or either, bubbles in the inferior mandibular cortex;

- Low width of the inferior mandibular cortex;

- Trabecular disorganization, low number and low connectivity;

- Accented contrast between mandibular ramus/body and structures of reinforcement, as the oblique line;

- Accented radiolucide of the jaws.

1.3 - Evidence against using panoramic radiographs to screen for osteoporosis. A trial was conducted to determine whether radiographic changes could be detected in the mandible of patients with mild to moderate postmenopausal osteoporosis and whether these changes could be used as a diagnostic tool to differentiate normal from osteoporotic patients. Subjects were classified as either osteoporotic ( $\mathrm{n}=$ $21)$ or normal $(n=14)$ on the basis of BMD of the lumbar spine and femoral neck, as determined by DEXA. Mandibular BMD measurements were made on panoramic and periapical radiographs and expressed in terms of millimeters of aluminum equivalent. There were no significant differences in any of the mandibular measurements between the normal and osteoporotic subjects. Whereas the skeletal bone measurements were correlated with each other, there was no correlation between skeletal and mandibular bone measurements. Women with mild to moderate osteoporosis could not be distinguished from women with normal bone density (Mohajery \& Brooks, 1992).

PMI was used in a group of postmenopausal women to determine whether it correlates with BMD of the femoral neck, lumbar area, and the trabecular and cortical parts of the mandible (Klemetti et al., 1993). BMD measured by DEXA for the femoral neck and lumbar area and by quantitative computed tomography (QCT) for the mandible. Linear correlation of the PMI with all BMD values was weak. However, the low and high index subgroup means were clearly dependent on the BMD variables. It was concluded that despite significant differences in PMI between osteoporotic subjects and controls, panoramic assessment should not be advocated as an assessment for osteoporosis (Otogoto \& Ota, 2003). 
It was investigated whether osteoporotic postmenopausal women show a decrease in mandibular cortical -bone height, as measured by the PMI index, when compared with non-osteoporotic post-menopausal women. Seventytwo Caucasian females ( 33 cases $/ 39$ controls), age range 5471 years, were selected through records and screening via a DEXA. ANOVA test indicated no differences in the mean PMI between case and control groups ( 0.37 to 0.15 and 0.38 \pm 0.13 , respectively; $\mathrm{p}=0.69$ ).

1.4. Osteoporosis and periodontal disease. Studies have also suggested that osteoporosis and periodontitis are associated diseases (Persson et al., 2002). Persson et al., investigated the prevalence of self-reported history of osteoporosis in an older, ethnically diverse population; (Farman et al.) the agreement between panoramic and mandibular cortical index (MCI) findings and self-reported osteoporosis; and (Watanabe et al., 2004a) the likelihood of having both a self-reported history of osteoporosis and a diagnosis of periodontitis. Panoramic radiographs and medical histories were obtained from 1.084 female Chinese subjects aged 60-75 years (mean age $68 \pm 5$ years). Subjects were graded either as not having periodontitis or with one of three severity grades of periodontitis. A positive MCI was found in $39 \%$ of the subjects, in contrast to $8 \%$ self-reported OP. The intra-class correlation between MCI and self-reported osteoporosis was $0.20(\mathrm{p}<0.01)$. The likelihood of an association between osteoporosis and MCI was 3\% (95\% CI: 1.6, 4.1, p < 0.001). Subjects with self-reported osteoporosis and a positive MCI had worse periodontal conditions $(p<0.01)$. The prevalence of positive MCI was high and consistent with epidemiological studies, but only partly consistent with a self-reported history of osteoporosis with a higher prevalence of positive MCI.Horizontal alveolar bone loss was associated with both positive self-reported osteoporosis and MCI findings. Contrary findings were find by authors (30) that examined the periodontal conditions in an age cohort of 70-year-old women comparing an osteoporosis group with a control group with normal BMD ( 210 women 70 years old). BMD of the hip was measured by DEXA. Nineteen women were diagnosed as having osteoporosis (BMD $<0.640 \mathrm{~g} / \mathrm{cm} 2$ in total hip) and 15 of them agreed to participate in the study. As a control group 21 women with normal bone mineral density (BMD $>0.881$ $\mathrm{g} / \mathrm{cm} 2$ ) were randomly selected from the initial population. The examination included a panoramic radiograph and vertical bitewings. The subjects completed a questionnaire on general health, age at menopause, concurrent medication, smoking and oral hygiene habits. No statistically significant differences in gingival bleeding, probing pocket depths, gingival recession and marginal bone level were found between the women with osteoporosis and those with normal bone mineral density. In conclusion, the study revealed no statistically significant differences in periodontal conditions or marginal bone level between the two groups; however, these results must be interpreted with caution since the compared groups were small.

It was studied the correlation between periodontal disease and osteoporosis by comparing age, panoramic radiographic and clinical parameters of periodontal disease. Diagnosis of osteoporosis in periodontal diseased patients was evaluated by panoramic radiographic parameters (mandibular cortical width MCW). Subjects were untreated adults with periodontal disease who were free of other systemic disease and who 20 or more teeth. They were examined by panoramic radiography recording alveolar bone loss (ABL), mandibular bone mass with the use of mandibular cortical width (MCW). ABL was significantly higher and MCW significantly lower postmenopausal subjects ( $>6$ years after menopause). The number of teeth was significantly lower in the postmenopausal group ( $>11$ years after menopause). Age and ABL correlated positively in men and women. Women whose MCW was less than mean (- 2 SD) should be diagnosed as osteoporotic. The results demonstrated that periodontal disease correlates with osteoporosis. MCW could be useful in detecting of osteoporosis in women with periodontal disease (Otogoto $\&$ Ota).

Some authors (Jagelavic`iene`\& Kubilius, 2006) evaluated the relationship between general osteoporosis of the organism and periodontal diseases. Radiological examination is informative in determining the type and the degree of alveolar resorption, the condition of the peridontium, and the number of teeth. These parameters provide valuable information when searching for correspondence and the correlation of data in studies.

2. Diabetes Mellitus. Diabetes mellitus is a common disorder of carbohydrate metabolism through either decreased production of insulin or tissue resistance to the effects of insulin (Mohammad et al.). The former (Type-1 diabetes) is insulin-dependent; the latter (Type-2 diabetes) is non-insulindependent and primarily treated by dietary modification.

The hypothesis that the risk for alveolar bone loss is greater, and bone loss progres-sion more severe, for subjects with poorly-controlled Type-2 diabetes mellitus compared toindividuals without Type-2 diabetes or with better controlled disease were test (Taylor et al., 1998). Of 359 subjects aged 15 to 57 with less than $25 \%$ radiographic bone loss at baseline, 338 did not have diabetes, 14 were better controlled diabetics, and 7 were poorly controlled diabetics. Panoramic radiographs were used to assess interproximal bone level. Bone scores (scale 0-4) corresponding to bone 
loss of $0 \%, 1 \%$ to $24 \%, 25 \%$ to $49 \%, 50 \%$ to $74 \%$, or $\geq 75 \%$ were used to identify the worst bone score in the dentition. Change in worst bone score at follow-up was specified on a 4 category ordinal scale as no change, or a 1,2,3 or 4 category increases over baseline. Poorly controlled diabetes, age, calculus, time to follow-up examination, and initial worst bone score were statistically significant explanatory variables in ordinal logistic regression models. Poorly controlled Type-2 diabetes mellitus was positively associated with greater risk for a change in bone score (compared to subjects without diabetes). The cumulative odds ratio (COR) at each threshold of the ordered response was $11(95 \% \mathrm{CI}=2.5$, 53.3). When contrasted with subjects with better-controlled diabetes, the COR for those in the poorly controlled group was $5(95 \% \mathrm{Cl}=0.8,53.3)$. The COR for subjects with better controlled diabetes was $2(95 \% \mathrm{CI}=0.7,6.5)$, when contrasted to those without diabetes. These results suggest that poorer glycemic control leads to both an increased risk for alveolar bone loss and more severe progression over those without Type-2 diabetes mellitus. There may also be a gradient, with the risk for bone loss and more severe progression over those without Type- 2 intermediate between those for poorly-controlled diabetes and non-diabetics.

People with Type-2 diabetes mellitus were disproportionately at risk of experiencing stroke, because hyperglycemia and other risk factors associated with diabetes accelerate development of cervical carotid artery atheromas. Removal of these atheromas may reduce the incidence of stroke. A study was (Friedlander et al., 2002) conducted a study to ascertain if those treated without insulin (non-insulin-treated, or NIT) would have a lower prevalence of atheromas on their radiographs and a lower prevalence of risk factors than those treated with insulin (insulin-treated, or IT). They evaluated the panoramic radiographs and medical records of 46 neurologically asymptomatic men $(n=34)$ and women $(n=12)$ (age range 62-77 years, mean age 68.5 years) with Type 2-diabetes. The panoramic radiographs showed that $24 \%$ of the NIT patients and $36 \%$ of the IT patients had atheromas; this difference was not statistically significant $(p=.52)$. The groups had similar risk factors that is, high levels of glycosylated hemoglobin A, or HbA1c; smoking; hypertension; and obesity ( $\mathrm{p}>.05)$. When compared with the $4 \%$ atheroma prevalence rate among healthy people of similar age, the rates were significantly higher in both the NIT $(p=.02)$ and IT $(p=.0006)$ patients. These results demonstrate that persons with Type-2 diabetes, irrespective of treatment modality, have high rates of atheroma as visualized on their panoramic radiographs. So, dentists treating patients with Type-2 diabetes mellitus should review their panoramic radiographs carefully for evidence of atheroma formation, because the modification of atherogenic risk factors and the surgical removal of atheromas in certain people have been shown to reduce the likelihood of stroke.

The mandibular bone mineral density was assessed in patients with Type 2 diabetes mellitus using panoramic radiographs. Nineteen subjects with Type-2 diabetes mellitus and 17 control subjects participated in the study. Bone mineral density measurements were performed on the panoramic radiographs with the help of a five-step copper stepwedge phantom, attached to each film cassette, which was calibrated before hand by DEXA. The results showed mean mandibular BMD ( $\mathrm{g} \mathrm{cm}-2$ ) was $1.53 \pm 0.27$ in women and $1.52 \pm 0.29$ in men with Type- 2 diabetes. In age and sex matched control subjects the values were $1.56 \pm 0.28$ and $1.46 \pm 0.23$ in women and in men, respectively. No statistically significant difference was observed between groups in mandibular BMD. This study showed that bone mineral density of the mandible does not seem to be affected in patients with Type- 2 diabetes mellitus. It was observed that a panoramic radiograph could serve for accurate mandibular BMD determination, when calibrated well against DEXA.

Chronic periodontitis (CP) is associated with stroke and subclinical atherosclerosis, but clinical measurement of $\mathrm{CP}$ can be time consuming and invasive. The panoramic radiographically was assessed and studied if the $\mathrm{CP}$ is associated with nonstenotic carotid artery plaque as an ultrasound measure of subclinical atherosclerosis (Engebretson et al., 2005). Panoramic radiographs were obtained from 203 stroke-free subjects ages 54 to 94 years, during the baseline examination of the Oral Infections and Vascular Disease Epidemiology Study (INVEST). CP among dentate subjects was defined either categorically (periodontal bone loss $50 \%$ severe versus $<50 \%$ bone loss) or via tertile formation (for dose-response investigation), with edentulous subjects categorized separately. In all subjects, highresolution B-mode carotid ultrasound was performed. Carotid plaque thickness (CPT) and prevalence (present/absent) were recorded. Among dentate subjects with severe periodontal bone loss, mean CPT was significantly greater $(1.20 \pm 1.00$ $\mathrm{mm}$ versus $0.73 \pm 0.89 \mathrm{~mm} ; \mathrm{P}=0.003)$. CPT increased with more severe bone loss (upper versus lower tertile bone loss; $\mathrm{P}=0.049$; adjusted for age, sex, and hypertension). This apparent dose-response effect was more evident among never-smokers. In a fully adjusted multivariate logistic regression model, severe periodontal bone loss was associated with a nearly 4-fold increase in risk for the presence of carotid artery plaque (adjusted odds ratio, 3.64; CI, 1.37 to 9.65). Severe periodontal bone loss is associated independently with carotid atherosclerosis. Panoramic oral radiographs may thus provide an efficient means to assess $\mathrm{CP}$ in studies of atherosclerosis risk. 
3. Hyperparathyroidism. Primary hyperparathyroidism is relatively rare and results from an excess secretion of parathyroid hormones due to a hormone producing benign or malignant neoplasm, conform several authors (Morano et al., 2000; Neville et al., 1995). Most persons with primary hyperparathyroidism are over age 60 years. Women are more commonly affected than men (Scutellari et al., 1996). Secondary

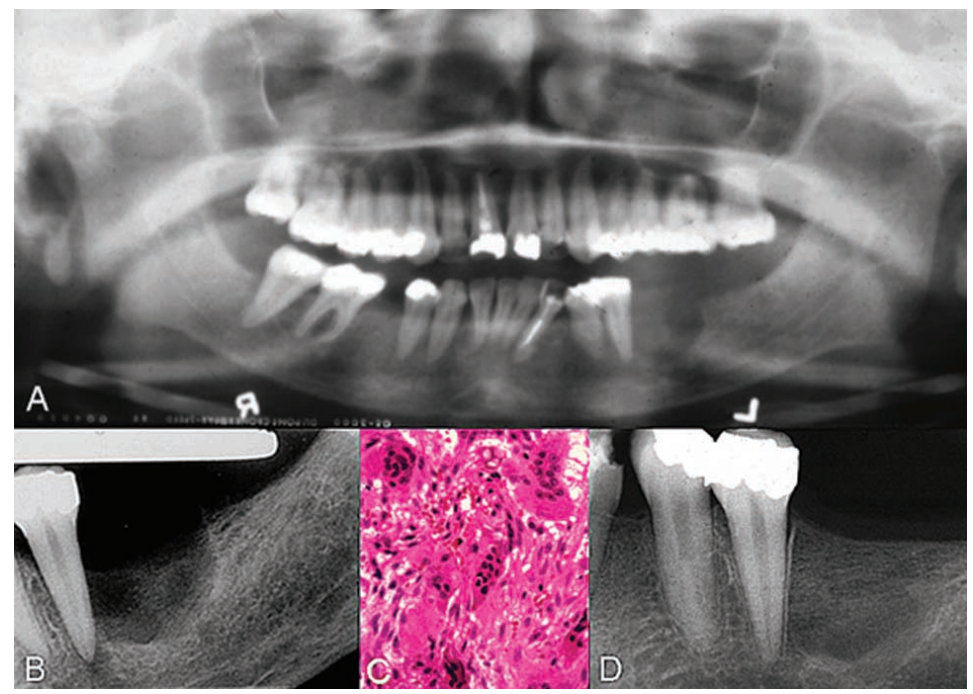

Fig. 4. Primary hyperparathyroidism - A. Panoramic radiograph demonstrating unilocular cystic lesion distal to the left mandibular second premolar. B. Periapical radiograph showing loss of lamina dura distal to the left mandibular second premolar tooth. C. Histopathologic study of the Brown tumor showing numerous multinucleated giant cells. D. The lesion healed and the lamina dura reconstituted following removal of the parathyroid tumor.

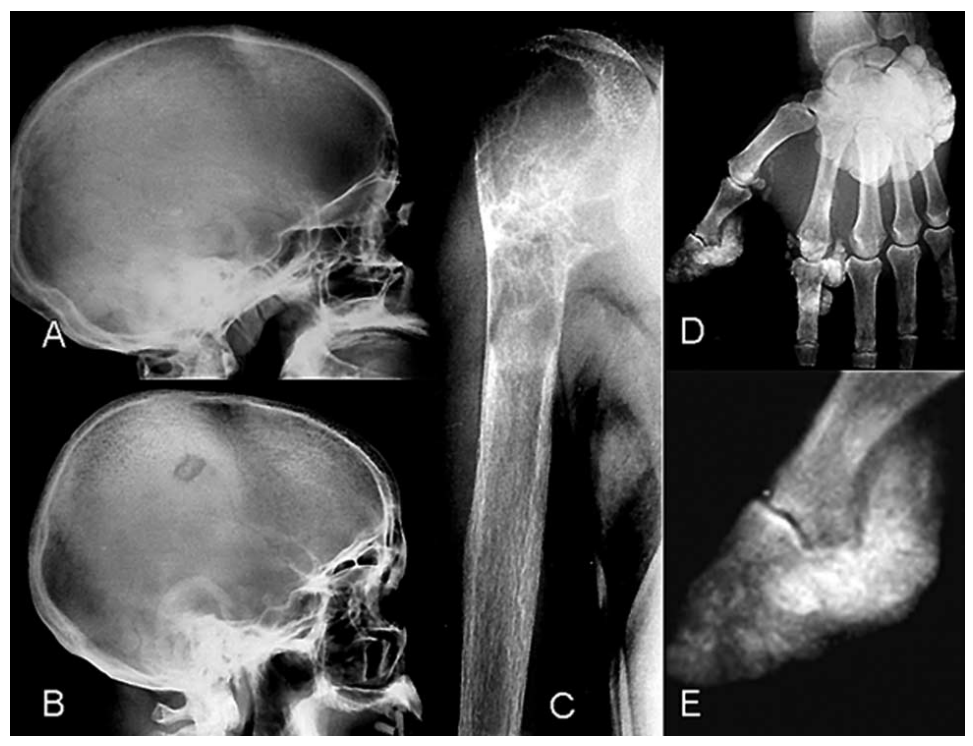

Fig.5. Hyperparathyroidism - A. Granular appearance of skull in patient having renal osteodystrophy. B. Solitary "punched-out" radiolucency in calvarium represents a Brown tumor in secondary hyperparathyroidism. C. Right humerus shows coarse internal trabeculation in primary hyperparathyroidism (same case as shown in Fig. 2). D. Metastatic calcifications in hand and wrist of patient with primary hyperparathyroidism. E. Detail of calcifications adjacent to thumb (detail of 2.D). hyperparathyroidism results in excess secretion of parathyroid hormone due to parathyroid hyperplasia compensating for a metabolic disorder that has resulted in retention of phosphate or depletion of the serum calcium level (Ganibegovic, 2000). The radiologic features of both forms of hyperparathyroidism are similar. These include generalized osteoporosis, unilocular or multilocular cystic radiolucencies in bone (Brown tumor), attenuation or loss of lamina dura surrounding the teeth, and calcifications in muscles and subcutaneous tissues (Figs. 4 and 5). It is often considered that histopathologic study of a biopsy specimen is the basis for diagnosis of "cystic" lesions of the jaws. Unfortunately, the Brown tumor provides no definitive histologic answer. Nuclear medicine or serologic confirmation is usually needed.

In Italy, 45 patients afflicted with chronic renal failure (29 men and 16 women; mean age: 48 years) and on haemodialysis for 4 to 245 months (mean: 67 months) were examined using panoramic images plus radiographs of the skull, hands, shoulders and clavicles, pelvis and spine (Ganibegovic). The control group (45 subjects with no renal diseases) was examined only by panoramic radiography. Dental and skeletal radiographs were rated on a 0-6 score and compared to assess possible relationships between skeletal and dental radiographic changes. Twenty-six dialysis patients (58\% of all dialysis patients studied) had the following radiographic abnormalities in the jaws: osteoporosis (100\%), lamina dura reduction or loss (27\%), calcifications of soft tissues or salivary glands (15\%), focal osteosclerosis adjacent to tooth roots (12\%), and Brown tumors (8\%). Radiographic abnormalities in the hand, shoulder and pelvis were found in $51 \%$ of dialysis patients. In the control group, only $16 \%$ had jaw lesions including osteopenia, cortex reduction at the mandibular angles and cystic lesions. It was concluded that panoramic radiography is useful in monitoring renal osteodystrophy, especially to assess the response to therapy such as parathyroidectomy or renal transplantation.

A study of panoramic and periapical radiographs of 42 patients on haemodialysis and having renal osteodystrophy, demonstrated a progressive increase in periodontal disease, loss of lamina dura, deviation in the trabecular pattern, Brown tumor "pseudocyst" formation and pulp calcifications (Bandeira et al., 2006). 
Bandeira et al., tell the Brazilian experience of the severe and soft primary hyperparathyroidism in Pernambuco-Brazil. The authors cited that in severe disease pathological fractures are frequently seen, especially in long bones of the lower extremities, and also loss of lamina dura of the teeth and salt-and-pepper appearance of the skull. At authors' institution, including outpatients and inpatients, the prevalence in postmenopausal women is $1.3 \%$.

4. Specific Infections. Not all systemic conditions that produce jaw lesions are as common as the ones discussed above, but their detection is equally important for the correct treatment to be commenced. In the developed world there had been a decline in advanced lesions from specific infections; however, with a growing population of immune-compromised individuals as a result of the more widespread use of immunosuppressive regimens subsequent to organ transplantation, and through the AIDS epidemic, a resurgence of previously "vanquished" organisms is possible.

4.1. Tuberculosis. Tuberculosis is a specific infection caused by the acid-fast bacillus Mycobacterium tuberculosis. Almost al cases arise from pulmonary disease. Involvement of the oral tissues is rare, occurring in less than one in 50 with tuberculosis (Farman et $a l$.). Oral tissues are involved through direct inoculation, extension from other infection sites, or haematogenous seeding. Patients with jawbone lesions complain of repeated attacks of "toothache-like" pain and there is usually swelling of the affected area. Sinus tracts develop as the swellings rupture and may drain intraorally or extraorally. Trismus may be present, especially if the temporomandibular joint is involved. Lesions within the jaws (Fig. 4) can be rarefactions with ill defined borders. There may be periosteal new bone formation. Sequestration of necrotic bone can occur. In addition to tuberculous osteomyelitis, calcified lymph upper cervical nodes from tuberculosis may also be detected on panoramic radiographs.

4.2. Syphilis. Syphilis is caused by infection with the spirochete Treponema pallidum. It may be congenital or acquired after birth. The acquired form can be subclassified into three distinctive stages: primary, secondary and tertiary. Bone may be affected in congenital syphilis and in both the secondary and tertiary stages of acquired syphilis (Fig. 5 ). The jaws are rarely affected by syphilis. When they are the palate is more frequently involved than the mandible. Radiographic features of bone involvement by syphilis include: deposition of subperiosteal new bone along the inferior border of the mandible (syphilitic periostitis); gummatous destruction of bone, especially the palate, resulting in a large radiolucent area; well demarcated destruction along a cortical margin; or multiple radiolucencies with poorly defined margins and sequestration (syphilitic osteomyelitis).

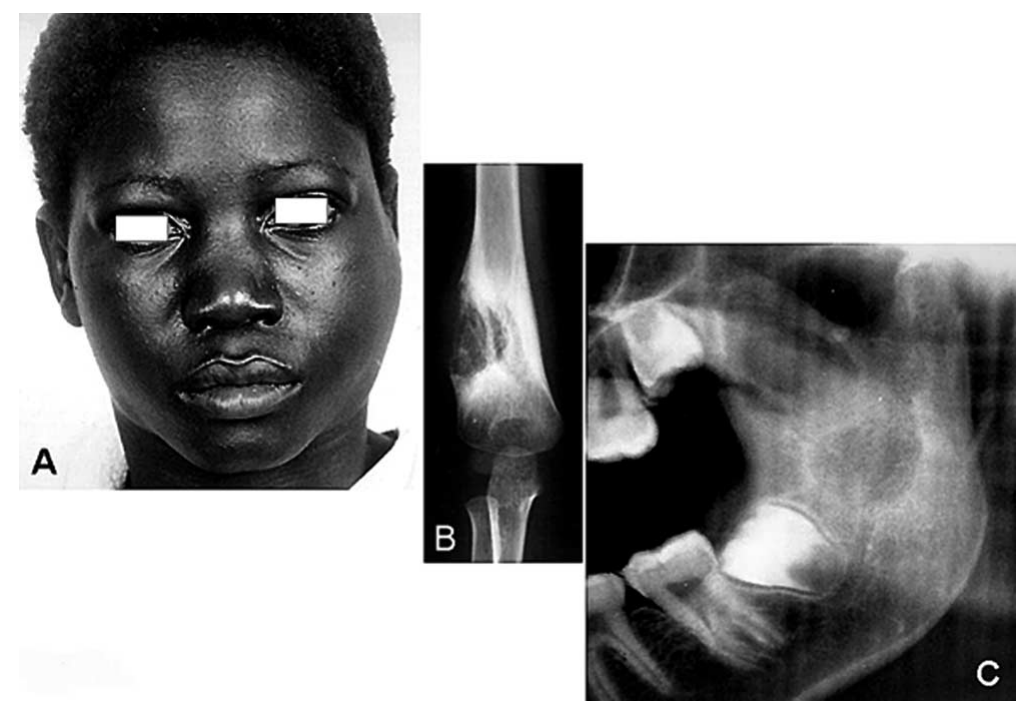

Fig. 6: Tuberculous osteomyelitis - A. Facial swelling is a frequent feature of this uncommon presentation of tuberculosis. B. Tuberculous osteomyelitis of long bone causing loss of cortical continuity. C. Detail from panoramic radiograph shows irregular radiolucency below the mandibular notch (tuberculous osteomyelitis).

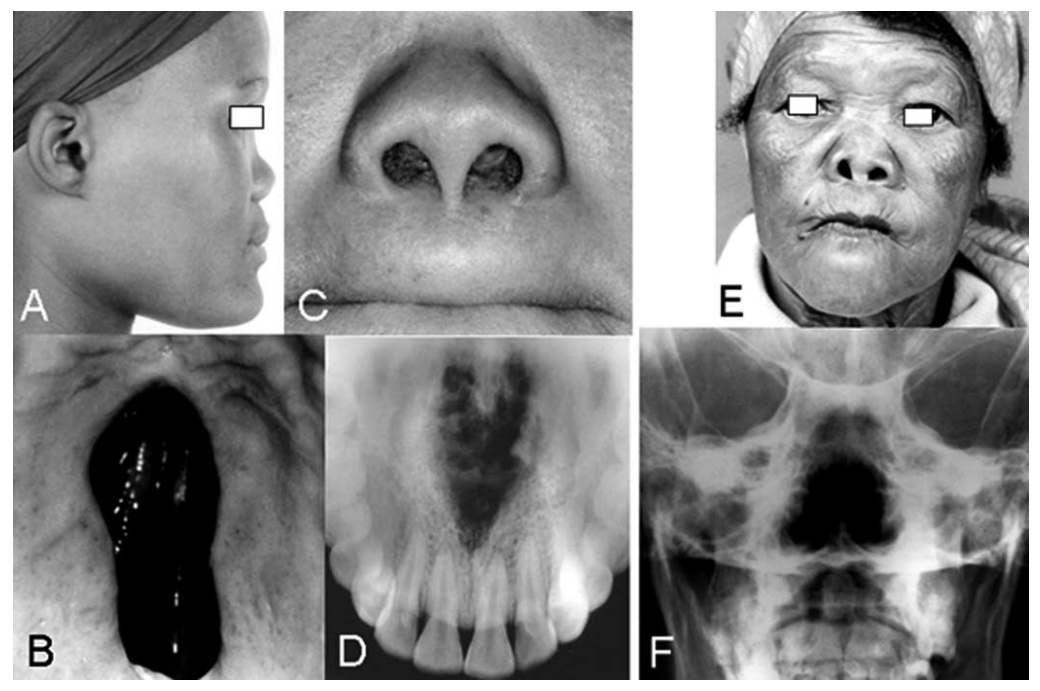

Fig. 7: A-D. Congenital syphilis. (Note deficient bridge of nose.) Lytic lesions in the center of the palate are outside the panoramic focal trough. E-F. Tertiary syphilis. (Note gummatous destruction in nasal cavity.) 
5. Metastatic Malignancies. Metastatic tumours to the jaws are rarely reported; however, metastases may well constitute the most common malignant tumours affecting the skeleton (Farman et al.). Nevertheless, most metastases to bone are found in the spine, pelvis, skull, ribs, or the humerus. It is reported that approximately one per cent of malignant neoplasms metastasize to the jaws, and metastases comprise about $1 \%$ of all oral malignancies. To qualify as a metastasis, the lesion must be localized to bone as distinguished from direct invasion and it should be histopathologically verifiable as a metastasis. Most metastases occur in mature individuals over age 50 years. The process of metastasis occurs by one of three routes: seeding of an adjacent body cavity, lymphatic spread or hematogenous dissemination. The most common primary sites for tumors metastasizing to the jaws in adults are from organs below the clavicle, namely: breast, kidney, lung, colon, rectum, prostate, stomach, skin, testes, bladder, ovary, and cervix. Above the clavicle, the most frequent primary site for metastases to the jaw is the thyroid gland. In children metastatic disease is extremely rare.

The clinical presentation of metastatic disease to the jaws is nonspecific, including local pain, swelling, numbness, paresthesia of the lip and chin, and loosening or extrusion of the teeth. Pathologic fractures may also occur but are considered rare (Fig. 6). The cardinal radiographic signs of metastases to the jaw include a well circumscribed but uncorticated lytic lesion, especially in the posterior mandible, with highly irregular outline, or multiple small areas of bone destruction that gradually coalesce to form large ill-defined areas of bone destruction (Figs. 6 and 7). Ancillary signs include periapical or periradicular radiolucency or radiopacity without evidence of pulpal pathology, failure of an extraction socket to heal, generalized loss of the lamina dura, or "floating" teeth. In a 12-month period, cancer metastatic to the mandible was diagnosed in eight patients at the Oral and Maxillofacial Surgery Clinic of the University of Vienna (Glaser et al., 1997). Six of them were presented with pain mimicking toothache, temporomandibular joint disorders, or trigeminal neuralgia, and two showed osteopenic bone lesions on panoramic radiography combined with perimandibular swelling. Histology revealed breast, lung, renal cancer, and a malignancy of inconclusive origin.

Thirty metastases of malignant tumors in jaws were retrospectively studied in the Pathology Department of a hospital in Paris, France (Auriol et al., 1991). They occurred more often in women than in men (17 F:13 M). In 21 cases, the primary cancer was known and had been treated one to four years earlier. In the other nine cases, discovery of the bone metastasis led to thediscovery of a latent tumor. Clinical signs and symptoms included swelling, pain, loosening of teeth, and labiomental anesthesia, but rarely pathologic fracture. All but two patients had a radiolucent lesion. The metastases almost always involved the mandible (95\%), most often in the molar area or angle. Histologically the majority of lesions were adenocarcinomas from breast (33\%) and alimentary canal (stomach, colon). Epidermoid bronchial carcinomas were seen in five cases and malignant melanomas in two cases. Only one sarcoma was involved, and this was from a liposarcoma of the thigh. In all but one patient, the disease was lethal over the short run (Figs. 8 and 9).

Concluding Remarks. While some controversy remains concerning the value of using panoramic radiographs in the screening of systemic diseases, the dentist should be capable of detecting features of such conditions when they produce changes on panoramic radiographs. Such conditions can have a major impact on the quality of life of afflicted patients. Early detection can lead to appropriate treatment and alleviation of untoward side affects. This is an area where the dentist may well save a life, valuing its performance as professional of health, understanding the patient as a whole.

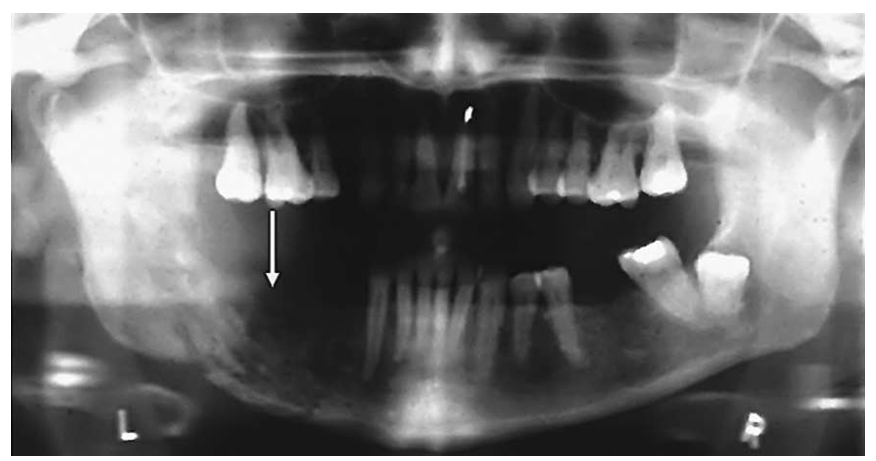

Fig. 8: Breast cancer metastasis to left mandibular body. Note "motheaten" appearance of the lesion and an associated pathological fracture.

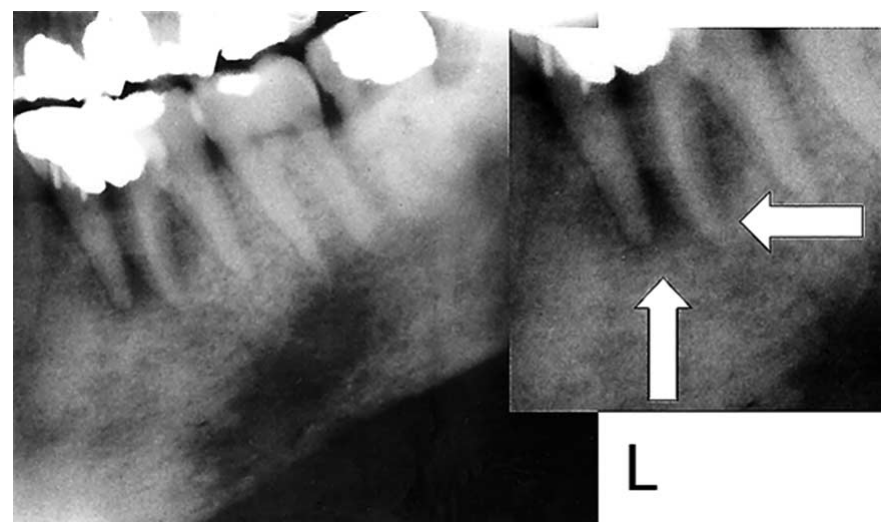

Fig. 9: Metastatic carcinoma. Note irregular "motheaten" rarefaction adjacent to first molar and second premolar teeth (detail from panoramic radiograph). Using the narrower perspective of a periapical radiograph, this lesion could well be misinterpreted as a simple "endo-perio" case. 
WATANABE, P. C. A.; FARMAN, A.; WATANABE, M. G. D. C. \& MARDEGAN ISSA, J. P. M. Detección de signos radiográficos en enfermedades sistémicas. Radiografía ortopantomográfica. Int. J. Morphol., 26(4):915-926, 2008.

RESUMEN: Par los propósitos de este informe, "enfermedad sistémica" puede ser interpretado como las condiciones que se producen dentro del cuerpo, más que estrictamente en los tejidos de la cavidad oral. Aunque habría que ocupar muchos volúmenes para revisar todas las condiciones, la intención de los autores sólo fue revisar algunos ejemplos de éstas, en las que la radiografía panorámica inicial, indica los resultados de la enfermedad generalizada, dada la suficiente importancia al afectar la calidad de vida y la longevidad del paciente.

PALABRAS CLAVE: Radiografía panorámica; Enfermedad sistémica.

\section{REFERENCES}

American Dental Association; U.S. Food \& Drug Administration. The Selection of Patients For Dental Radiograph examinations. Guidelines for prescribing dental radiographs, 2004.

Araújo, D. V.; Oliveira, J. H. A. \& Bracco, O. L. Custo da fratura osteoporótica de fêmur no sistema suplementar de saúde brasileiro. Arq. Brás. Endocrinol. Metab., 49(6):897-901, 2006.

Auriol, M.; Chomette, G.; Wann, A. \& Guibert, F. Metastases of malignant tumor in the jaw. Analysis of 30 case reports. Rev. Stomatol. Chir. Maxillofac., 92:155-9, 1991.

Bandeira, F.; Griz, L.; Caldas, G.; Bandeira, C. \& Freese, E. From mild to severe primary hyperparathyroidism: The Brazilian experience. Arq. Brás. Endocrinol. Metab., 50(4):657-3, 2006.

Benson, B. W.; Prihoda, T. J.; Glass, B. J. Variations in adult cortical bone mass as measured by a panoramic mandibular index. Oral. Surg. Oral. Med. Oral. Pathol., 71:349-56, 1991.

Bras, J.; Ooij, C. P.; van, Abraham-Inpijn K.; Kusen, G. J. \& Wilmink, J. M. Interpretation of the mandibular angular cortex: a diagnostic tool in metabolic bone loss. Part I. Normal state and postmenopausal osteoporosis. Oral. Surg., 53:541-5, 1982.

Bulgarelli, A. F.; Watanabe, P. C. A.; Silva, A. B. M.; Paulino, S. M. \& Pardini, L. C. Cisto de Retenção do Seio Maxilar - Considerações para o Clinico Geral. Rev. APCD., 56(3):178-81, 2002.

Engebretson, S. P.; Lamster, I.; Elkind, M. S. V.; Rundek, T.; Serman, N. J. \& Demmer, R. T. Radiographic Measures of Chronic Periodontitis and Carotid Artery Plaque. Stroke, 36:561-5, 2005.
Farman, A. G.; Nortjé, C, J. \& Wood, R. E. Oral and maxillofacial diagnostic imaging. Mosby -Year Book, St. Louis, 1993.

Friedlander, A. H.; Garrett, N. R. \& Norman, D.C. The prevalence of calcified carotid artery atheromas on the panoramic radiographs of patients with type 2 diabetes mellitus. J. Am. Dent. Assoc., 133(11):1516-23, 2002.

Ganibegovic, M. Dental radiographic changes in chronic renal diseases. Med. Arh., 54:115-8, 2000.

Glaser, C.; Lang, S.; Pruckmayer, M.; Mi Mesi, W.; Rasse, M.; Marosi, C. \& Leitira, T. Clinical manifestations and diagnostic approach to metastatic cancer of the mandible. Int. J. Oral. Maxillofac. Surg., 26:365-8, 1997.

Homer, K.; Devlin, H. \& Harvey, L. Detecting patients with low skeletal bone mass. J. Dent., 30:171-5, 2002.

Jagelavic`iene`, E. \& Kubilius, R. The relationship between general osteoporosis of the organism and periodontal diseases. Medicina, 42(8):613-8, 2006.

Klemetti, E.; Komakov, S.; Heiskanen, P.; Vainio, P. \& Lassila, V. Panoramic mandibular index and bone mineral densities in postmenopausal women. Oral. Surg. Oral. Med. Oral. Pathol., 75:774-9, 1993.

Klemetti, E.; Kolmakov, S. \& Kröger H. Pantomography in assessment of the osteoporosis risk group. Scand. J. Dent. Res., 102:68-72, 1994.

Knezovic'-Zlataric', D. \& C`elebic', A. Comparison of mandibular bone density and radiomorphometric indices in wearers of complete or removable partial dentures. Oral. Radiol., 21(2):51-5, 2005. 
Marques Neto, J. F. \& Lederman, R. Osteoporose: Brasil. $1^{\text {st }}$ ed. Limay, São Paulo, 1995.

Mohajery, M. \& Brooks, S. L. Oral radiographs in the detection of early signs of osteoporosis. Oral. Surg. Oral. Med. Oral. Pathol., 73:112-7, 1992.

Mohammad, A. R.; Alder, M. \& McNally, M. A. A pilot study of panoramic film density at selected sites in the mandible to predict osteoporosis. Int. J. Prosthodont., 9:290-4, 1996.

Morano, S.; Cipriani, R.; Gabriele, A.; Medici, F. \& Pantelini, F. Recurrent Brown tumors as initial manifestation of primary hyperparathyroidism. An unusual presentation. Minerv. Med., 91:117-22, 2000.

Nakamoto, T.; Taguchi, A.; Ohtsuka, M.; Suei, Y.; Fujiya, M. \& Tanimoto, K. Dental panoramic radiograph as a tool to detect postmenopausal women with low bone mineral density: untrained general dental practitioners' diagnostic performance. Osteoporos. Int., 14:659-64, 2003.

Neville, B. W.; Damm, D. D.; Allen, C. M. \& Bouquot, J. E. Oral and Maxilofacial Pathology. EB Saunders Company, Philadelphia, 1995.

Otogoto, J. \& Ota, N. Correlation between periodontal disease and osteoporosis using panoramic radiographic parameters for diagnosed osteoporosis in dental clinic. Clin. Calcium., 13(5):582-6, 2003.

Persson, R. E.; Hollender, L. G.; Powell, L. V.; MacEntee, M. I.; Wyatt, C. C. \& Kiyak, H. A. Assessment of periodontal conditions and systemic disease in older subjects. I. Focus on osteoporosis. J. Clin. Pedodontol., 29(9):796-802, 2002.

Scutellari, P. N.; Orzinxblo, C.; Bedani, P. L. \& Romano, C. Radiographic manifestations in teeth and jaws in chronic kidney insufficiency. Radial. Mad., 92:415-20, 1996.

Secretaria da Saúde. Hospital do Servidor Público Estadual. Osteoporosis 1995: basic diagnosis and therapeutic elements for a National Consensus Proposal. São Paulo Med. J. 113:1-64, 1995.

Secretaria de Vigilância Sanitária; Ministério da Saúde Brasil. Portaria 453 - "Diretrizes de proteção radiológica e radiodiagnóstico médico e odontológico” Brasília (Brasil), 1998.
Taguchi, A.; Suei, Y.; Sanada, M.; Higashi, Y.; Ohtsuka, M. \& Nakamoto, T. Detection of Vascular Disease Risk in Women by Panoramic Radiography. J. Dent. Res., 82(10):838-43, 2003.

Taguchi, A.; Suei, Y.; Sanada, M.; Ohtsuka, M.; Nakamoto, T. \& Sumida, H. Validation of Dental Panoramic Radiography Measures for Identifying postmenopausal Women with Spinal Osteoporosis. A. J. R., 183:175560, 2004

Taylor, G. W.; Burt, B. A.; Becker, M. P.; Genco, R. J. \& Shlossman, M. Glycaemia control and alveolar bone loss progression in type 2 diabetes. Ann. Periodontol., 3:3039-43, 1998 .

Watanabe, P. C. A. Relação de Três Indicadores de Qualidade Óssea na Pesquisa da Osteoporose em Radiografias Panorâmicas. Tese de apresentada para a obtenção do Título de Livre-docente da USP, Ribeirão Preto, 2003.

Watanabe, P. C. A.; Arita, E. S.; Monteiro, S. A. C.; Oliveira, T. M. \& Taguchi, A. The relationship among three indicators of bone quality in the osteoporosis research on panoramic radiographic. Osteoporos. Int., 15(1):S67-255, 2004a.

Watanabe, P. C. A.; Monteiro, S. A. C.; Lacerda, S. A.; Taguchi, A. \& Arita, E. S. Interrelation of two quality indicators for osteoporosis research in panoramic XRays of Brazilian Women. Rev. Int. Estomatol., 1(3):200-6, 2004b.

White, S. C.; Atchison, K. A.; Gornbein, J. A.; Nattiv, A.; Paganini-Hill, A. \& Service, S. K. Change in mandibular trabecular pattern and hip fracture rate in elderly women. Dentomaxillofac. Radiol., 34(3):168$74,2005 a$

White, S. C.; Taguchi, A.; Kao, D.; Wu, S.; Service, S. K. \& Yoon, D. Clinical and panoramic predictors of femur bone mineral density. Osteoporos. Int., 16(3):339-46, $2005 b$.

White, S. C.; Taguchi, A.; Kao, D.; Wu, S.; Susan, K. S.; Yoon, D.; Suei, Y.; Nakamoto, T. \& Tanimoto, K. Clinical and panoramic predictors of femur bone mineral density. International Osteoporosis Foundation; National osteoporosis Foundation, 2004.

Wowern, N. Bone mass of mandibles. In vitro and in vivo analyses (Thesis). Danish Med. Bull., 33:23-44, 1986. 
WATANABE, P. C. A.; FARMAN, A.; WATANABE, M. G. D. C. \& ISSA, J. P. M. Radiographic signals detection of systemic disease. Orthopantomographic radiography. Int. J. Morphol., 26(4):915-926, 2008

Wowern, N. General and oral aspects of osteoporosis: a review. Clin. Oral. Invest., 5:71-82, 2001.

Correspondence to:

Dr. Plauto C. A. Watanabe

Faculdade de Odontologia de

Ribeirão Preto - USP

Av. do Café s/n.

CEP:14040-904

Ribeirão Preto-SP.

BRASIL

Email: watanabe@forp.usp.br

Received: 27-05-2008

Accepted: 18-09-2008 